\title{
MRI-guided stereotactic radiofrequency thermocoagulation for 100 hypothalamic hamartomas
}

\author{
Shigeki Kameyama, MD, PhD, ${ }^{1}$ Hiroshi Shirozu, MD, PhD, ${ }^{1}$ Hiroshi Masuda, MD, ${ }^{1}$ Yosuke Ito, MD, ${ }^{1}$ \\ Masaki Sonoda, MD,' and Kohei Akazawa, PhD² \\ ${ }^{1}$ Hypothalamic Hamartoma Center, Department of Functional Neurosurgery, Nishi-Niigata Chuo National Hospital; and \\ ${ }^{2}$ Department of Medical Informatics, Niigata University Medical and Dental Hospital, Niigata, Japan
}

OBJECTIVE The aim of this study was to elucidate the invasiveness, effectiveness, and feasibility of MRI-guided stereotactic radiofrequency thermocoagulation (SRT) for hypothalamic hamartoma $(\mathrm{HH})$.

METHODS The authors examined the clinical records of 100 consecutive patients (66 male and 34 female) with intractable gelastic seizures (GS) caused by HH, who underwent SRT as a sole surgical treatment between 1997 and 2013. The median duration of follow-up was 3 years (range 1-17 years). Seventy cases involved pediatric patients. Ninety percent of patients also had other types of seizures (non-GS). The maximum diameter of the HHs ranged from 5 to 80 $\mathrm{mm}$ (median $15 \mathrm{~mm}$ ), and 15 of the tumors were giant HHs with a diameter of $30 \mathrm{~mm}$ or more. Comorbidities included precocious puberty (33.0\%), behavioral disorder (49.0\%), and mental retardation (50.0\%).

RESULTS A total of 140 SRT procedures were performed. There was no adaptive restriction for the giant or the subtype of $\mathrm{HH}$, regardless of any prior history of surgical treatment or comorbidities. Patients in this case series exhibited delayed precocious puberty $(9.0 \%)$, pituitary dysfunction $(2.0 \%)$, and weight gain $(7.0 \%)$, besides the transient hypothalamic symptoms after SRT. Freedom from GS was achieved in $86.0 \%$ of patients, freedom from other types of seizures in $78.9 \%$, and freedom from all seizures in $71.0 \%$. Repeat surgeries were not effective for non-GS. Seizure freedom led to disappearance of behavioral disorders and to intellectual improvement.

CONCLUSIONS The present SRT procedure is a minimally invasive and highly effective surgical procedure without adaptive limitations. SRT involves only a single surgical procedure appropriate for all forms of epileptogenic $\mathrm{HH}$ and should be considered in patients with an early history of GS.

http://thejns.org/doi/abs/10.3171/2015.4.JNS1582

KEY WORDS epileptic encephalopathy; gelastic seizure; hypothalamic hamartoma; outcome; stereotactic radiofrequency thermocoagulation; surgical treatment; epilepsy

$\mathrm{H}$ YPOTHALAMIC hamartoma $(\mathrm{HH})$ is a rare congenital abnormality, which presents with a hallmark feature of intractable gelastic seizures (GS). There is now strong evidence that $\mathrm{HH}$ involves intrinsic epileptogenesis, ${ }^{7-12,14,17}$ and surgical treatment of $\mathrm{HH}$ itself is considered important for seizure control. Although various surgical treatments are available for epileptic patients with $\mathrm{HH}$, the selection of treatment modalities depends on the type and size of the HH, as well as the surgeons' preference. ${ }^{3}$ Mittal et al. reported that no single neurosurgical approach is like- ly to treat all forms of $\mathrm{HH}$, and that a multimodal or staged procedure must be used to treat the lesion. ${ }^{16}$ Similarly, the Barrow group commonly use staged and multiple surgical approaches for $\mathrm{HH}$ treatment, including microsurgical resection, endoscopic disconnection, and stereotactic radiosurgery. ${ }^{6,19,24}$ We previously developed a surgical procedure involving MRI-guided stereotactic radiofrequency thermocoagulation (SRT) of HH and reported an early series of cases in which this technique was used. ${ }^{9,12}$ In the present study, which was undertaken to determine the invasiveness

ABBREVIATIONS DQ = developmental quotient; EEG = electroencephalography; EFP = emotional facial paresis; FIQ = full-scale IQ; GS = gelastic seizures; HH = hypothalamic hamartoma; SISCOM = subtraction ictal SPECT co-registered to MRI; SMA = supplementary motor area; SPECT = single-photon emission computed tomography; SRT = stereotactic radiofrequency thermocoagulation; WAIS = Wechsler Adult Intelligence Scale; WAIS-R = WAIS-Revised; WAIS-III = WAIS-Third Edition; WISC = Wechsler Intelligence Scale for Children; WISC-R = WISC-Revised; WISC-III = WISC-Third Edition.

ACCOMPANYING EDITORIAL See pp 1501-1502. DOI: 10.3171/2015.5.JNS15785.

SUBMITTED January 12, 2015. ACCEPTED April 6, 2015.

INCLUDE WHEN CITING Published online November 20, 2015; DOI: 10.3171/2015.4.JNS1582. 
and feasibility of SRT for all forms of HH without surgical restriction, we retrospectively reviewed the surgical outcomes of 100 cases in which patients underwent SRT as a sole surgical treatment in our hospital. Although Parvizi et al. ${ }^{18}$ previously reported a neuroanatomical analysis of $100 \mathrm{HH}$ lesions in terms of seizures and comorbidities, to our knowledge, this is the first report on seizure outcomes after a single surgical procedure applied to a large number of $\mathrm{HH}$ cases from a single institution.

\section{Methods}

All patients or their parents gave written informed consent before the presurgical evaluation and surgical treatment.

\section{Patient Profile}

We retrospectively reviewed 100 consecutive patients (66 male and 34 female) who underwent SRT of HHs between October 1997 and September 2013. The patients' ages at surgery ranged from 1 to 50 years (median 10.0 years); 70 patients were younger than 16 years of age (pediatric group). Of the 100 cases, there was a clinical history of precocious puberty in 33 cases and behavioral disorder in 49. There was no sex bias in the prevalence of precocious puberty. Patients were followed up for 1-17 years (median 3 years) after surgery for evidence of postoperative seizures, functional outcomes, and surgical complications.

There were 6 cases of Pallister-Hall syndrome (positive GLI3 gene mutation in 5). There was no familial HH. The duration of epilepsy prior to the present surgery ranged from 0 to 47 years (median 7.1 years). All patients had intractable GS, with the age of onset ranging from neonatal $(\mathrm{n}=30)$ and infantile $(\mathrm{n}=18)$ to 11 years (median 1.0 year). Ten patients had GS alone. According to patient history, 90 patients had various other types of seizures -complex partial seizures $(n=59)$, secondarily generalized tonic-clonic seizures $(n=51)$, tonic seizures $(n=36)$, atonic seizures $(n=10)$, myoclonic seizures $(n=3)$, and epileptic spasm $(n=3)$-with age of onset ranging from 0 to 21 years (median 4.0 years). We interviewed the patients and their family members about the level of awareness and emotional feeling at the ictal onset, and reviewed ictal video-electroencephalography (EEG) data to analyze ictal seizure semiology.

Twenty-nine patients underwent various types of prior treatments including microsurgery $(\mathrm{n}=14)$, radiosurgery $(n=9)$, and multimodal surgeries $(n=6)$ at other institutes. Multimodal surgical treatment included microsurgery and radiosurgery in 3 cases; endoscopic surgery and radiosurgery in 1 case; microsurgery, endoscopic surgery, and radiosurgery in 1 case; and microsurgery, radiosurgery, and SRT in 1 case. Of 3 patients with severe motor and intellectual disabilities, one underwent multimodal surgical treatment (triple microsurgeries, radiosurgery, and SRT), one underwent radiosurgery, and one had multiple brain abnormalities in addition to $\mathrm{HH}$ lesion due to PallisterHall syndrome.

\section{Examination}

We obtained ictal video-electroencephalograms during a 3-day study in all but 2 patients because of relatively frequent seizures. The presence of laterality of facial expression and EEG findings were used to help determine the approach side for SRT. Although patients or their parents reported seizure and functional outcomes, we confirmed seizure freedom on postoperative video-EEG and behavioral improvement in follow-up studies. Endocrinological examination without a stimulation test was performed at presurgical evaluation and postoperative follow-up. A neuropsychologist evaluated pre- and postoperative intellectual and behavioral condition. Full-scale IQ was evaluated using the Wechsler Intelligence Scale for ChildrenRevised (WISC-R) or Third Edition (WISC-III), or with the Wechsler Adult Intelligence Scale-Revised (WAIS-R) or Third Edition (WAIS-III). Developmental quotient (DQ) was evaluated using the Enjoji Child Development Test or the Tanaka-Binet Intelligence Scale. Interictal and ictal SPECT was performed using ${ }^{99} \mathrm{~m}$ Tc-ethylcysteinate dimer. In 85 patients, subtraction ictal SPECT co-registered to MRI (SISCOM) also provided information on the ictogenic area within the $\mathrm{HH}(\mathrm{n}=36)$ and the epileptic propagation to the hemisphere $(n=61)$.

\section{Clinical Characteristics}

We confirmed awareness during GS in 29 patients, laughing with vocalization in 59 patients, and asymmetrical facial expression during GS in 53 patients on preoperative video-EEG. We classified our patients into pediatric and adult groups (Table 1). These 2 groups had significant differences in age at onset of non-gelastic seizures $(\mathrm{p}<$ $0.001)$ and duration of GS history $(\mathrm{p}<0.001)$. The pediatric group had significantly larger HHs $(\mathrm{p}=0.003)$ and a significantly higher prevalence of behavioral disorder $(\mathrm{p}<$ 0.001 ) than the adult group. Patients with behavioral disorder had a significantly larger lesion size than those without behavioral disorder and those with precocious puberty had a significantly larger lesion size than those without precocious puberty ( $\mathrm{p}<0.001$ for both comparisons). Moreover, patients with behavioral disorder had a significantly lower full-scale IQ (FIQ) or DQ than patients without behavioral disorder $(\mathrm{p}=0.015)$. Cognitive delay was reported in 50 patients (FIQ or DQ < 70).

This series included 15 patients (14 pediatric, 1 adult; 9 female, 6 male) with a giant $\mathrm{HH} \geq 30 \mathrm{~mm}$ in diameter (range 30-80 mm, mean $39 \mathrm{~mm}$ ). All giant HHs were of the mixed hypothalamic type, and 12 had bilateral attachment. Patients with giant HHs had a significantly higher prevalence of precocious puberty than the patients with smaller (non-giant) HHs $(73.3 \%, \mathrm{p}<0.001)$. No volume or any subtype of $\mathrm{HH}$ was excluded from SRT.

\section{MRI Classification}

We routinely examined axial, coronal, sagittal, and 3D MRI using T1-weighted, T2-weighted, and FLAIR sequences. For SRT, the $100 \mathrm{HHs}$ were classified into 4 subtypes (Table 2) on the basis of coronal MR images, as previously reported..$^{12}$ The lesions were classified into intrahypothalamic type with medial extension into the third ventricle, parahypothalamic type with inferior extension into the interpeduncular fossa without involvement of the 
TABLE 1. Summary of demographic and clinical characteristics of 100 pediatric and adult patients with $\mathrm{HH}$

\begin{tabular}{|c|c|c|c|c|}
\hline Characteristic & All Patients $(n=100)$ & Pediatric Group $(n=70)$ & Adult Group $(n=30)$ & p Value $†$ \\
\hline Female, n (\%) & $34(34.0)$ & $22(31.4)$ & $12(40.0)$ & $0.273^{\star *}$ \\
\hline Age at surgery in yrs, median (range) & $10.0(1-50)$ & $6.5(1-15)$ & $27.5(16-50)$ & - \\
\hline Onset of GS in yrs, median (range) & $1.0(0-11)$ & $0.8(0-10)$ & $1.0(0-11)$ & $0.531^{*}$ \\
\hline Onset of nongelastic Sz in yrs, median (range) & $4.0(0-21)$ & $2.5(0-13)$ & $11.0(3-21)$ & $<0.001^{*}$ \\
\hline GS history in yrs, median (range) & $7.1(0-47)$ & $5.0(0-15)$ & $27.0(5-47)$ & $<0.001^{*}$ \\
\hline GS only, n (\%) & $10(10.0)$ & $9(12.9)$ & $1(3.3)$ & $0.136^{* *}$ \\
\hline Max diameter of $\mathrm{HH}$ in mm, median (range) & $15.0(5-80)$ & $18.0(5-80)$ & $11.0(8-30)$ & $0.003^{*}$ \\
\hline \multicolumn{5}{|l|}{ MRI classification, n (\%) } \\
\hline Intrahypothalamic type & $25(25.0)$ & $13(18.6)$ & $12(40.0)$ & $0.024^{* *}$ \\
\hline Mixed hypothalamic type w/ bilateral attachment & $36(36.0)$ & $31(44.3)$ & $5(16.7)$ & $0.007^{* *}$ \\
\hline Mixed hypothalamic type w/ unilateral attachment & $33(33.0)$ & $23(32.9)$ & $10(33.3)$ & $0.082^{* *}$ \\
\hline Parahypothalamic type & $6(6.0)$ & $3(4.3)$ & $3(10.0)$ & $0.251^{* *}$ \\
\hline \multicolumn{5}{|l|}{ Comorbidities, $n(\%)$} \\
\hline Mental retardation & $50(50.0)$ & $35(50.0)$ & $15(50.0)$ & $0.586^{* *}$ \\
\hline Behavioral disorder & $49(49.0)$ & $43(61.4)$ & $6(20.0)$ & $<0.001^{* *}$ \\
\hline Precocious puberty & $33(33.0)$ & $25(35.7)$ & $8(26.7)$ & $0.260^{* *}$ \\
\hline
\end{tabular}

$\mathrm{GS}=$ gelastic seizure; $\mathrm{HH}=$ hypothalamic hamartoma,; $\mathrm{Sz}=$ seizures.

$\dagger$ For difference between the pediatric and adult groups.

* Mann-Whitney U-test.

** Fisher exact test.

third ventricle, and mixed hypothalamic type with a mixture of the above features with medial and inferior extension. The mixed hypothalamic type was further divided on the basis of attachment (bilateral or unilateral).

The maximum diameter of the HHs ranged from 5 to $80 \mathrm{~mm}$ (median $15.0 \mathrm{~mm}$ ). There was a significantly negative correlation between $\mathrm{HH}$ size and FIQ/DQ (mean 69.8, $\mathrm{r}=-0.172, \mathrm{p}=0.034)$, but not between $\mathrm{HH}$ size and age of GS onset. Table 2 summarizes the relationship between the subtypes of $\mathrm{HH}$ and the patients' clinical characteristics. All HHs were classified into intrahypothalamic type $(n=25)$, parahypothalamic type $(n=6)$, and mixed hypothalamic type $(n=69)$. Within the mixed hypothalamic type, there were $36 \mathrm{HH}$ with bilateral attachment and 33 with unilateral attachment. In this classification, the extension of $\mathrm{HH}$, especially pure intra- or extra-third ventricular extension, was reevaluated rather than the volume of the entire HH. The mixed hypothalamic type was characterized by a combination of medial extension and inferior extension. Unilateral attachment was divided into rightsided $(n=32)$ and left-sided $(n=32)$. Three HHs of the mixed hypothalamic type showed cystic formation within the lesion. The HHs of the intrahypothalamic type were significantly smaller (median $10.0 \mathrm{~mm}, \mathrm{p}<0.05$ ) than the HHs of the mixed hypothalamic type with bilateral attachment (median $22.5 \mathrm{~mm}$ ) or unilateral attachment (median $14.0 \mathrm{~mm}$ ) or the parahypothalamic type (median 11.5 $\mathrm{mm})$. The preoperative FIQ or DQ in patients with lesions

TABLE 2. MRI classification and clinical characteristics

\begin{tabular}{lcccc}
\hline \multicolumn{1}{c}{ Variable } & Intrahypothalamic Type & $\begin{array}{c}\text { Mixed Hypothalamic Type } \\
\text { w/ Bilateral Attachment }\end{array}$ & $\begin{array}{c}\text { Mixed Hypothalamic Type } \\
\text { w/ Unilateral Attachment }\end{array}$ & Parahypothalamic Type \\
\hline No of cases (\%) & $25(25.0)$ & $36(36.0)$ & $33(33.0)$ & $6(6.0)$ \\
\hline Attachment & & & & \\
\hline Bilateral & 0 & 36 & 14 & 0 \\
\hline Right & 16 & 0 & 19 & 2 \\
\hline Left & 9 & 0 & $14.0(8.0-35.0)^{*}$ & 4 \\
\hline HH size in mm, median (range) & $10.0(5.0-13.0)$ & $22.5(10.0-80.0)^{*}$ & $76.0(9-100)$ & $67.0(31-104)$ \\
\hline FIQ/DQ, median (range) & $86.0(40-127)$ & $59.5(10-107)^{*}$ & $15(45.5)$ & $3(50.0)$ \\
\hline Mental retardation, $n(\%)$ & $9(36.0)$ & $23(63.9)$ & $18(54.5) \dagger$ & $2(33.3)$ \\
\hline Behavioral disorder, $n(\%)$ & $5(20.0)$ & $24(66.7) \dagger$ & $9(27.3) \dagger$ & $3(50.0) \dagger$ \\
\hline Precocious puberty, $n(\%)$ & $0(0.0)$ & $21(58.3) \dagger$ & & \\
\hline
\end{tabular}

$D Q=$ development quotient; $F I Q=$ full-scale $I Q$.

* $p<0.05$ for comparison with intrahypothalamic type, Mann-Whitney U-test with Bonferroni correction for level of significance.

$\dagger p<0.05$ for comparison with intrahypothalamic type, chi-square for independence test with Bonferroni correction for level of significance. 
of the intrahypothalamic type (median 86.0) was significantly higher than that of patients with the mixed hypothalamic type with bilateral attachment $(59.5, \mathrm{p}<0.05)$. The patients with mixed hypothalamic type $\mathrm{HH}$ with bilateral or unilateral attachment had a significantly higher prevalence of behavioral disorder $(66.7 \%$ and $54.5 \%$, respectively) and precocious puberty $(58.3 \%$ and $27.3 \%$, respectively) than those with intrahypothalamic type $\mathrm{HH}$ $(\mathrm{p}<0.05)$. The patients with lesions of the parahypothalamic type also had a significantly higher prevalence of precocious puberty $(50.0 \%, \mathrm{p}<0.05)$. The adult group had a significantly higher number of intrahypothalamic type $\mathrm{HH}(40.0 \%, \mathrm{p}=0.024)$, whereas the pediatric group had a significantly higher number of mixed hypothalamic type with bilateral attachment $(44.3 \%, \mathrm{p}=0.007)($ Table 1$)$.

\section{Surgical Procedures}

The basic surgical procedures for MRI-guided SRT were previously reported. ${ }^{12}$ We decided on the side of the first SRT surgery according to the dominant attachment of HH, EEG dominancy of epileptic discharges, SISCOM findings, and the contralateral side to the lopsided grimace of asymmetrical smile during GS.

In this series, all patients underwent SRT (single or multiple procedures) as the sole surgical treatment for HH. All procedures were performed with local anesthesia using $1 \%$ lidocaine hydrochloride and general anesthesia using propofol and fentanyl/remifentanil. For the adult patients who could not accept general anesthesia, all procedures were performed with local anesthesia and total intravenous anesthesia using propofol. SRT surgery consisted of 3 steps.

\section{First Step}

MRI-guided stereotactic planning for the target localization was performed using the reconstructed multidimensional image planning software of the SurgiPlan workstation (Leksell SurgiPlan, Elekta), and unilateral frontal trajectories were determined (Fig. 1). 3D T1weighted and FLAIR images with the x-ray coordinate indicator were imported for the SurgiPlan analyses. In addition to axial images, the reconstructed images of coronal, sagittal, path's eye, arc parallel, and arc orthogonal views provided additional and useful information in determining the target and in avoiding ablation of normal brain tissue. We planned to make 1-6 spherical lesions of $5 \mathrm{~mm}$ diameter at each coordinate on a single trajectory within the HH. Multiple lesions and trajectories were planned in order to form an ideal disconnecting plane between the $\mathrm{HH}$ and the hypothalamus, depending on the localization and the area of the interface. We believe that ablation of the entire volume of the lesion unnecessary when this method is used. MRI-guided planning is helpful to avoid injury to nerves and vessels outside of the $\mathrm{HH}$.

\section{Second Step}

We performed stereotactic neurosurgery using the stereotactic multipurpose method (Elekta) and an originally designed and manufactured MicroManipulator (Elekta Japan) to place a probe on a target. Semi-microrecording was performed with a coaxial stereotactic electrode (tungsten tip, $10 \mu \mathrm{m}$ in diameter, $0.5-\mathrm{M} \Omega$ impedance; Unique Medical) using Neuromaster MEE1216 (Nihon Kohden) with the sampling frequency set at $1000 \mathrm{~Hz}$, to confirm the border of the $\mathrm{HH}$ and the localization of epileptic spikes within the $\mathrm{HH}$ on the first trajectory to the target. The stereotactic neurosurgical setting involved use of a radiofrequency thermocoagulation probe $(2 \mathrm{~mm}$ in diameter). The location error of the center of the probe tip (4 $\mathrm{mm}$ in length) is actually within $1 \mathrm{~mm}$. If we required more than 3 trajectories, 2 or 3 bur holes were prepared on the unilateral frontal cranium.

\section{Third Step}

After test heating $\left(60^{\circ} \mathrm{C}\right.$ for 30 seconds) to confirm safety, therapeutic thermocoagulation was performed by heating the target at $74^{\circ} \mathrm{C}$ for 60 seconds using the Leksell NeuroGenerator (Elekta) in order to create a 5-mm spherical lesion within the HH. This exact volume of the ablation was always confirmed by experimental thermocoagulation using the same parameters in egg albumin 1 week before SRT. The exact coagulation volume was controlled by precise heat control because radiofrequency heating does not affect the cerebrospinal fluid. During thermocoagulation, the patients' physiological condition (heart rate, blood pressure, blood oxygen saturation, and deep body temperature) was monitored. After creation of a single lesion, the thermocoagulation process was repeated to create additional lesions using the same trajectory unless only a single lesion was planned for that trajectory. Postoperative CT was always performed to confirm the coagulated volume as a low-density area within the HH without massive hemorrhage or unexpected edema immediately after surgery. Follow-up was routinely scheduled for 3 months, 1 year, 2 years, 3 years, and 5 years after SRT. We assessed the exact volume and localization of the ablation area on postoperative MRI at follow-up. If patients had residual or recurrent seizures and evidence of incomplete disconnection on MRI, we planned to perform an additional operation.

\section{Statistical Analysis}

All statistical analyses were performed using PASW Statistics for Windows, Version 18 (SPSS Inc.). Statistical significance was set at $p<0.05$. Fisher's exact and MannWhitney U-tests were used for comparison between the pediatric and adult groups. Spearman's rank correlation coefficient was used for a statistical correlation of the clinical characteristics between patients with and without comorbidities and between patients with and without residual seizures. Chi-square for independence and MannWhitney U tests with Bonferroni correction for level of significance were used for comparison between patients with the 4 different tumor types based on MRI classification. Postoperative changes of FIQ or DQ at the 1-year follow-up evaluation were evaluated by paired t-test.

\section{Results \\ SRT Procedures}

Intraoperative semi-microrecording confirmed the low level of electrical activity at the border of $64 \mathrm{HHs}$ and 


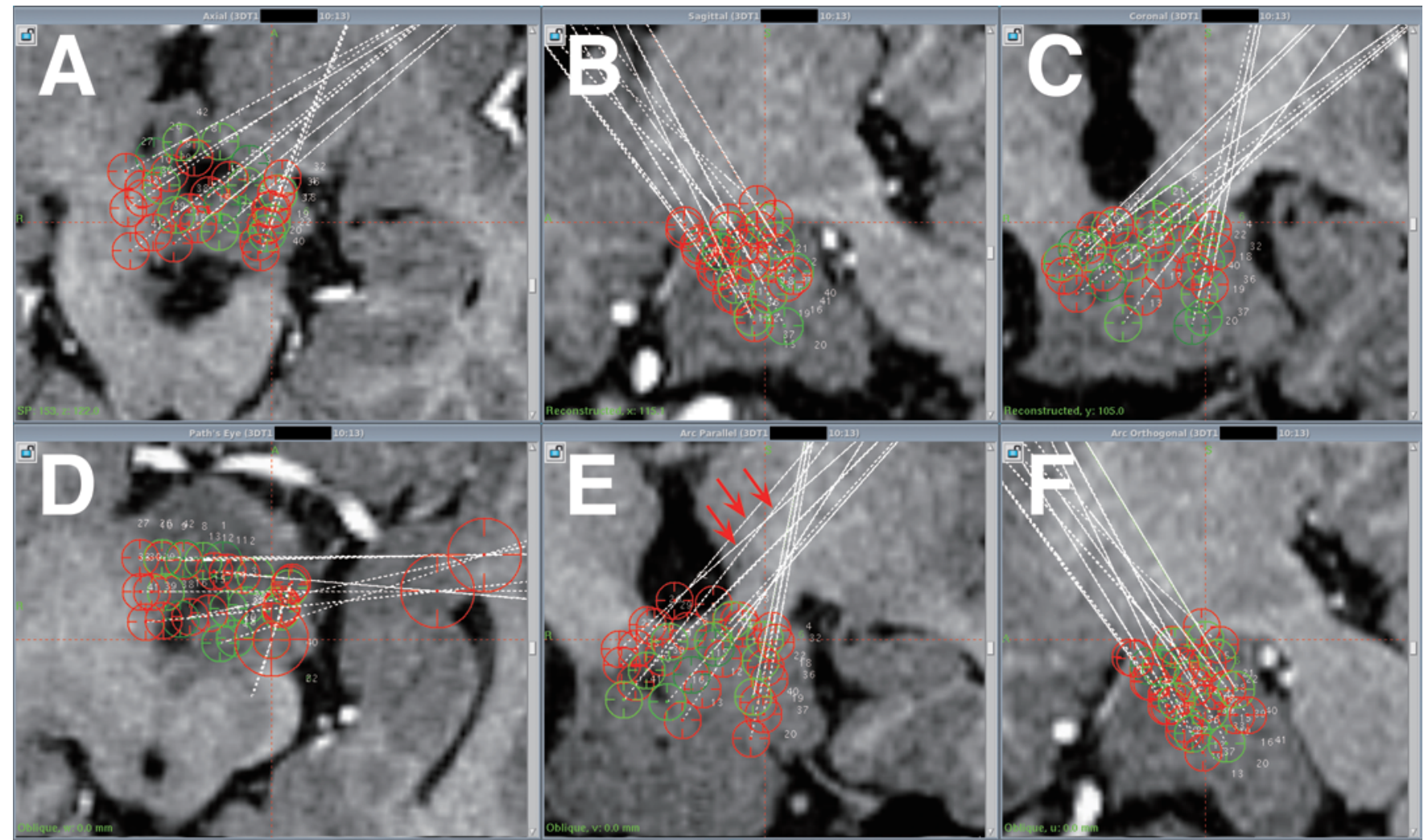

FIG. 1. Screenshot showing presurgical planning for SRT on the 6 planes used in this study. The 33 tentative targets and 10 trajectories from the 3 different entries for a giant mixed hypothalamic type of $\mathrm{HH}$ with bilateral attachment and a diameter of 43 $\mathrm{mm}$ are presented on T1-weighted MR images in the axial plane $(\mathbf{A})$ and in the following reconstructed planes: sagittal (B), coronal (C), path's eye (D), arc-parallel (E), and arc-orthogonal (F). Green or red circles show the planned targets, which were estimated as 5-mm-diameter spherical lesions. Red circles are located far from each real plane. The white dotted lines show the virtual paths to the targets. The intersection of the red dotted lines that run at right angles shows the coordinates of the center of the present target. Three trajectories were set by a trans-third ventricular approach to the contralateral attachment (red arrows in E).

the localization of epileptic spikes near the interface of $38 \mathrm{HHs}$. A total of 140 SRT procedures were performed, all via unilateral approaches (Table 3). Sixty-eight patients underwent a single SRT procedure and 32 patients needed multiple SRT procedures. There was no significant difference in SRT rates between pediatric and adult groups. SRT was used to create 1-36 coagulated lesions (median 6) via 1-10 trajectories (median 4) depending on the size of the $\mathrm{HH}$ and the area of attachment. The pediatric group received a median of 6 lesions per SRT procedure (total 103 SRTs, 73.6\%), which was not significantly different from the median of 4 lesions per SRT in the adult group (total 37 SRTs, 26.4\%). The pediatric group required significantly more trajectories than the adult group (median 4 vs $3, p=0.008$ ) because of significantly larger $\mathrm{HH}$ size (median $18.0 \mathrm{~mm}$ vs $11.0 \mathrm{~mm}, \mathrm{p}=0.003$ ). However, our SRT procedures were largely the same, despite the variation in the age of the patients and the size of the HHs.

\section{Repeat SRTs}

We attempted a staged bilateral approach for bilateral attachment in 6 of the 100 patients. The interval between the 2 surgeries ranged from 4 to 47 months (median 8 months). However, since 2013 we have attempted single-stage SRT with a unilateral approach for bilateral attachment of large and giant HHs in 9 patients to avoid bilateral damage to the hypothalamus. We developed this method involving a unilateral approach to the ipsilateral attachment and a transthird ventricular approach to the contralateral attachment in a single stage (Fig. 1E, red arrows, and Fig. 2) because we had observed 2 cases of pituitary dysfunction after the bilateral approach. In $\mathrm{HH}$ with bilateral attachment, there were 5 single SRTs with a successful trans-third ventricular approach, whereas 4 patients required repeat SRT surgeries. Repeat surgeries for residual and recurrent GS or non-GS are shown in the outcome-flow diagrams (Fig. 3). The repeat surgeries included 2 SRTs $(\mathrm{n}=27), 3$ SRTs $(\mathrm{n}=$ $2)$, and 4 SRTs $(n=3)($ Table 3$)$.

\section{Surgical Complications}

Immediate postoperative CT showed a low-density ablation area within the $\mathrm{HH}$ without unexpected edema or massive hemorrhage in all patients. Although postoperative MRI at around 2 weeks after surgery showed a slightly hyperintense area with various degrees of edema in the hamartoma, the attached hypothalamus, and around the trajectories, there was prolonged disturbance of consciousness in only 1 patient. The findings of edema always 
TABLE 3. Surgical procedures and results

\begin{tabular}{|c|c|c|c|c|}
\hline Variable & All Patients & Pediatric Group & Adult Group & $\mathrm{p}$ Value \\
\hline No. of cases $(\%)$ & $100(100.0)$ & $70(70.0)$ & $30(30.0)$ & \\
\hline Single SRT, n (\%) & $68(68.0)$ & $45(64.3)$ & $23(76.7)$ & $0.163^{*}$ \\
\hline Multiple SRTs, n (\%) & $32(32.0)$ & $25(35.7)$ & $7(23.3)$ & - \\
\hline 2 SRTs & $27(27.0)$ & $20(28.6)$ & $7(23.3)$ & - \\
\hline 3 SRTs & $2(2.0)$ & $2(2.9)$ & $0(0.0)$ & - \\
\hline 4 SRTs & $3(3.0)$ & $3(4.3)$ & $0(0.0)$ & - \\
\hline No. of trajectories, median (range) & $4(1-10)$ & $4(1-10)$ & $3(1-7)$ & $0.008^{* *}$ \\
\hline No. of lesions, median (range) & $6(1-36)$ & $6(1-36)$ & $4(1-14)$ & $0.132^{* *}$ \\
\hline \multicolumn{5}{|l|}{ Seizure outcome, n (\%) } \\
\hline GS freedom & $86(86.0)$ & $58(82.9)$ & $28(93.3)$ & $0.141^{*}$ \\
\hline Non-GS freedom $\ddagger$ & $71(78.9)$ & $51(83.6)$ & $20(69.0)$ & $0.096^{*}$ \\
\hline Overall seizure freedom & $71(71.0)$ & $51(72.8)$ & $20(66.7)$ & $0.346^{*}$ \\
\hline Withdrawal of antiepileptic drugs, $n(\%)$ & $18(18.0)$ & $14(20.0)$ & $4(13.3)$ & $0.312^{*}$ \\
\hline \multicolumn{5}{|l|}{ Postoperative hormonal disorder, $\mathrm{n}(\%)$} \\
\hline Delayed precocious puberty & $9(9.0)$ & $9(12.9)$ & - & - \\
\hline Weight gain & $7(7.0)$ & $5(7.1)$ & $2(6.7)$ & $0.650^{*}$ \\
\hline Pituitary dysfunction & $2(2.0)$ & $2(2.9)$ & $0(0.0)$ & $0.350^{*}$ \\
\hline No. of SRTs, n (\%) & $140(100.0)$ & $103(73.6)$ & $37(26.4)$ & - \\
\hline \multicolumn{5}{|l|}{ Transient surgical complication, $n(\%)$} \\
\hline Horner's syndrome & $84(60.0)$ & $66(64.1)$ & $18(48.6)$ & $0.075^{*}$ \\
\hline Emotional facial paresis & $77(55.0)$ & $61(59.2)$ & $16(43.2)$ & $0.069^{*}$ \\
\hline Hyperphagia & $39(27.9)$ & $28(27.2)$ & $11(29.7)$ & $0.461^{*}$ \\
\hline Hyperthermia & $31(22.1)$ & $29(28.2)$ & $2(5.4)$ & $0.002^{*}$ \\
\hline Hyponatremia & $31(22.1)$ & $24(23.3)$ & $7(18.9)$ & $0.382^{*}$ \\
\hline Short memory disturbance & $12(8.6)$ & $9(8.7)$ & $3(8.1)$ & $0.605^{*}$ \\
\hline Intracranial hemorrhage, asymptomatic & $4(2.9)$ & $4(3.9)$ & $0(0.0)$ & $0.288^{*}$ \\
\hline \multicolumn{5}{|c|}{$\begin{array}{l}\dagger \text { For difference between the pediatric and adult groups. } \\
\text { * Fisher exact test. } \\
\text { ** Mann-Whitney U-test. } \\
\text { ‡ Percentage represents proportion of the } 90 \text { patients with non-gelastic seizures (in addition to GS) who achieved freedom from those seizure } \\
\text { types. }\end{array}$} \\
\hline
\end{tabular}

resolved by the 3-month follow-up. There were 2 cases of postoperative pituitary dysfunction after repeat SRTs for bilateral attachment. One patient was treated with replacement therapy for low levels of cortisol and thyroid stimulating hormone. The second patient was treated with nasally administered desmopressin at bedtime for diabetes insipidus. Endocrine function was generally unchanged after SRT except for the patients with postoperative pituitary dysfunction. There were numerous other transient complications after the 140 SRT surgeries, including Horner's syndrome (after 84 SRTs, 60.0\%), hyperphagia (after 39, $27.9 \%$ ), hyponatremia $\leq 130 \mathrm{mEq} / \mathrm{L}$ with some medical treatment (after $31,22.1 \%$ ), hyperthermia $\geq 38.5^{\circ} \mathrm{C}$ (after $31,22.1 \%$ ), short-term memory disturbance (after 12 , $8.6 \%$ ), and prolonged disturbance of consciousness for 3 months (after $1,0.7 \%$ ) as hypothalamic symptoms, as well as emotional facial paresis (after 77, 55.0\%) or asymptomatic intracranial hemorrhage (after 4, 2.9\%) (Table 3). Although the prevalence of these transient complications was almost equal between the 2 groups, there was a significantly higher prevalence of hyperthermia in the pedi- atric group $(p=0.002)$. A higher prevalence of short-term memory disturbance was associated with the cases of bilaterally attached HH. Immediately after 35 SRTs, patients presented with obvious hypotonia, hypokinesia, and dysdiadochokinesis of the contralateral limbs, likely as transient supplementary motor symptoms. Emotional facial paresis (EFP) occurred exclusively on the contralateral side (not on the side treated by SRT), and volitional facial expression remained symmetrical in these patients. EFP always persisted longer than the duration of hypotonia and hypokinesia of the limbs. The recovery stage of EFP was variable in duration. This phenomenon persisted for several months to 1 year or more after SRT. Horner's syndrome, especially sweating disorder, was not always transient, and could continue for more than 1 year. Repeat SRTs did not increase the complication rate. During the follow-up period, precocious puberty developed in 9 patients and weight gain was observed in 7 patients.

\section{Seizure Outcomes}

Freedom from GS was achieved in $86.0 \%$ of cases and 
freedom from all seizures types in $71.0 \%$. Many patients (74.4\% of those who became GS free) achieved GS freedom immediately after SRT, while some had residual seizures that disappeared within 2 weeks $(9.3 \%), 2$ weeks to 3 months $(7.0 \%), 3$ months to 1 year $(5.8 \%)$, and after 1 year (3.5\%). All patients who achieved GS freedom had a follow-up MRI showing complete disconnection between the $\mathrm{HH}$ and the hypothalamus. In 90 patients with non-gelastic seizures, freedom from these seizure types was achieved in $78.9 \%$, including $30.0 \%$ with immediate freedom (Table $3)$. There was no difference in seizure outcomes between the 2 groups. The first SRT led to freedom from GS for $64(64.0 \%)$ of the 100 patients in this study and freedom from non-gelastic seizures in $71(78.9 \%)$ of the 90 patients who had other seizure types in addition to GS. Seizure outcomes for patients who had giant HHs and those with smaller (nongiant) HHs were also similar. Eighteen of the patients who achieved complete seizure freedom were able to maintain it after withdrawal of antiepileptic medication during the follow-up period.

Intervals between SRT to GS recurrence were variable-within 2 weeks (25.9\%), within 3 months $(31.5 \%)$, within 1 year $(9.3 \%)$, and after 1 year $(7.4 \%)$. Repeat SRTs were effective for residual GS but ineffective for residual seizures of other types (Fig. 3). Nineteen (21.1\%) of the 90 patients with nongelastic seizures who underwent repeat SRT had recurrent or residual nongelastic seizures within 1 year after the procedure. Although the patients with residual nongelastic seizures included 9 children, this group had a significantly longer duration of GS history $(p=0.03)$ than the group who achieved freedom from nongelastic seizures. Residual nongelastic seizures were mainly tonic seizures $(n=8)$, complex partial seizures $(n=7)$, epileptic spasms $(n=2)$, and myoclonic seizures $(n=1)$.

\section{Functional Outcomes}

The behavioral problems were resolved in all patients who achieved GS freedom. In the patients with residual GS or nongelastic seizures, behavioral improvement was also achieved. In this series, almost all patients achieved intellectual improvement to various degrees. The exceptions were patients with severe motor and intellectual disabilities. One-year follow-up FIQ/DQ were available for 69 patients; the mean score was 6.1 points higher than the mean preoperative score for this group of patients $(\mathrm{p}<$ $0.001)$

\section{Discussion}

\section{Surgery for Hypothalamic Hamartoma}

Since the discovery that $\mathrm{HH}$ involves intrinsic epileptogenesis, ${ }^{8,14,17}$ numerous surgical procedures have been developed. However, as HH lesions are located deep near the midline of the skull base, they are difficult to reach with microsurgical approaches, which are also too invasive to provide total resection. Therefore, minimally invasive surgical procedures, such as endoscopic surgery, radiosurgery, SRT, and stereotactic laser ablation (SLA), have been introduced. Endoscopic resection or disconnection with a trans-third ventricular approach is also a more elegant surgery. ${ }^{2,5,6,22}$ Delalande and Fohlen ${ }^{5}$ proposed the important

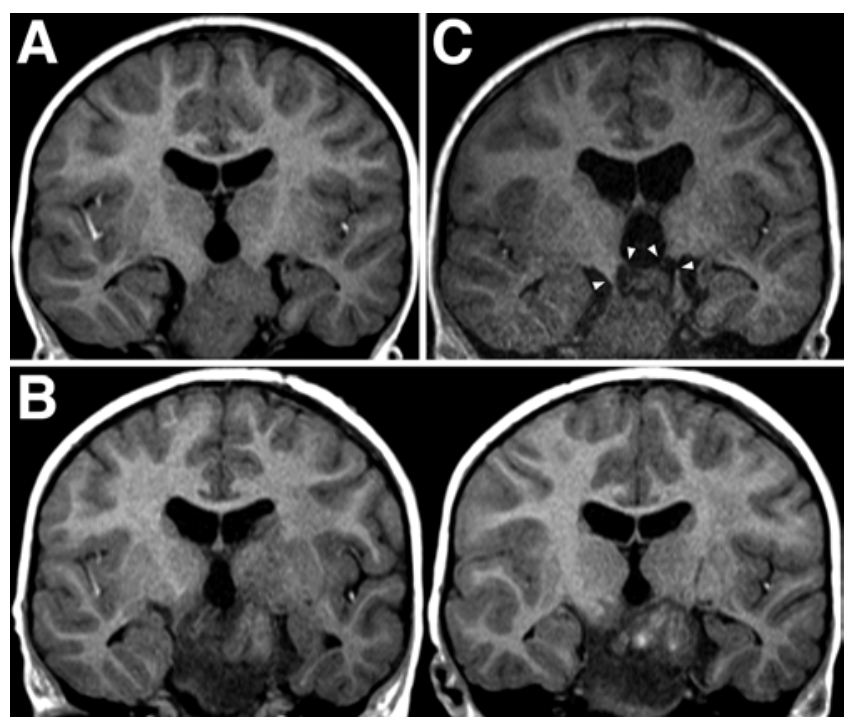

FIG. 2. Coronal T1-weighted MR images obtained in the same patient whose presurgical planning is shown in Fig. 1 showing sequential changes in the $\mathrm{HH}$. A: Preoperative image showing the giant mixed hypothalamic type of $\mathrm{HH}$ with bilateral attachment. B: Postoperative images obtained 2 weeks after SRT. C: Postoperative image (obtained 1 year after SRT) showing complete disconnection of the bilateral attachment (arrowheads). The lower half of the lesion was left intact. The patient had achieved seizure freedom at 1-year follow-up.

concept of disconnection between the lesion and the hypothalamus for preventing the direct propagation of GS. We agree with this concept, which was used as a basis for our SRT procedure. A recent report of thulium laser-based endoscopic disconnection demonstrated higher effectiveness with less invasiveness than disconnection with monopolar coagulation. ${ }^{2}$ However, there are limitations of endoscopic surgery for large HHs, as previously described by Rekate et al. ${ }^{22}$ Régis et al. ${ }^{21}$ reported that radiosurgery is much safer than microsurgical resection and should be regarded as the first-line option for small to middle-sized HHs. Radiosurgery can also have surgical limitations based on $\mathrm{HH}$ volume, and radiosurgical disconnection has never been advocated. ${ }^{21}$ In a report of SLA therapy, 12 (86\%) of the 14 patients achieved seizure freedom without permanent surgical complications. ${ }^{27}$ Although stereotactic procedures are minimally invasive and highly effective for small or medium-sized HHs, further studies are expected in terms of surgical indications and limitations, especially for the treatment of large or giant HHs.

Several reports have suggested that no single neurosurgical approach is likely to treat all forms of $\mathrm{HH} .{ }^{16,19,24} \mathrm{Nev}-$ ertheless, in our institute we have treated over 130 patients with $\mathrm{HH}$ by a single surgical strategy of SRT. As there are no reports of results from a single neurosurgical strategy for a large number of $\mathrm{HH}$ in a single institution, we retrospectively reviewed the outcomes from 100 consecutive patients treated at our institute. Our key findings were that our SRT procedure was minimally invasive and achieved good outcomes for all forms of the sessile type of $\mathrm{HH}$, without restriction based on patient age or $\mathrm{HH}$ volume (including giant $\mathrm{HH}$ ). Our SRT technique has never excluded patients who underwent prior surgery and/or radiosurgery. 


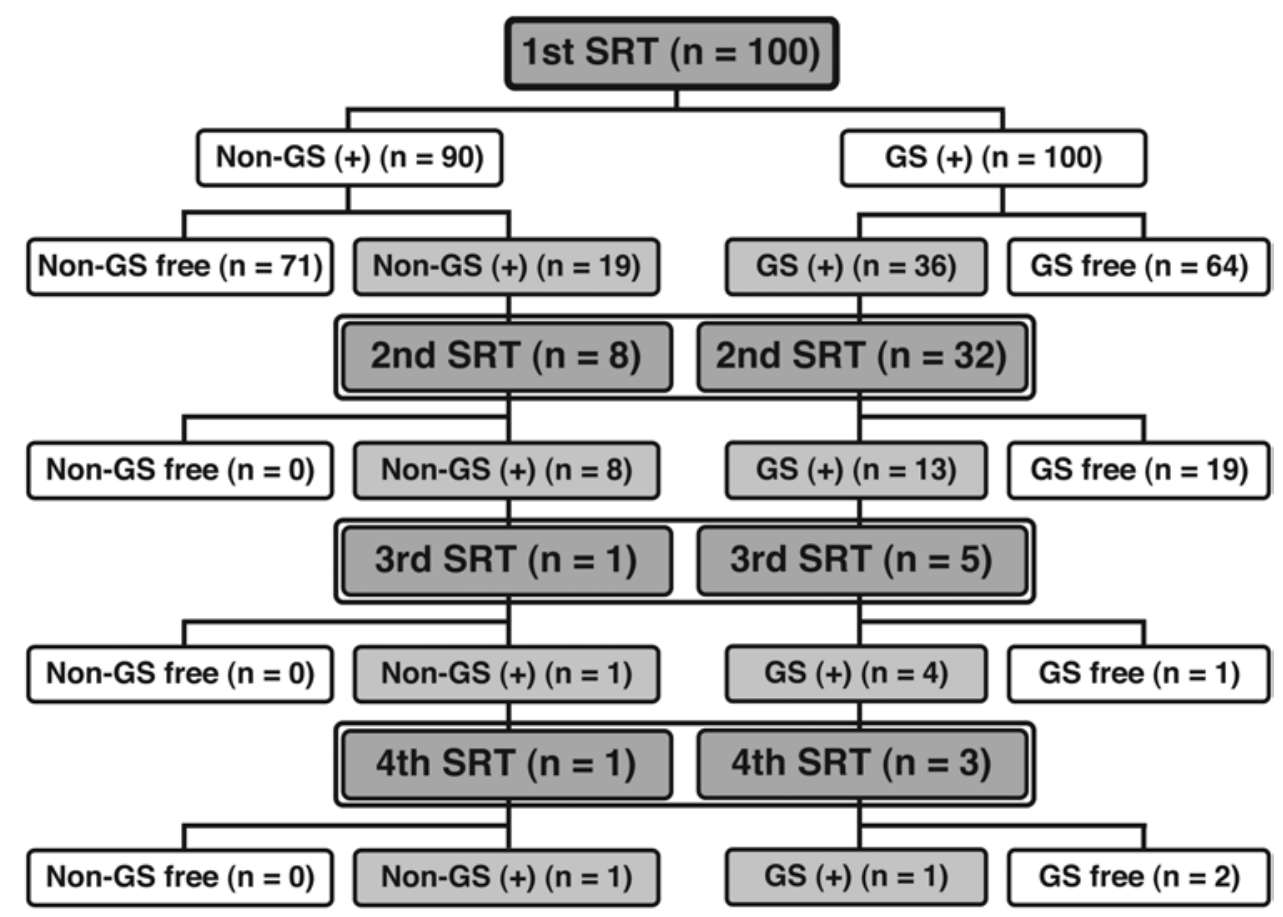

FIG. 3. Flow diagram showing the repeat surgeries and outcomes of SRT with respect to gelastic seizures (GS) and other types of seizures (non-GS) in 100 patients. Overall GS freedom and non-GS freedom were achieved in $86(86.0 \%)$ and $71(78.9 \%)$ patients, respectively. Five patients had a repeat SRT scheduled because of residual GS. Two patients were excluded from this evaluation because of short duration after the last repeat SRT.

Therefore, repeat SRT surgeries may also be undertaken at no extra risk if patients have residual or recurrent GS after the initial surgery.

The proposed rationale behind SRT for treatment of $\mathrm{HH}$ is as follows. The area of ictogenesis is located in the part of the $\mathrm{HH}$ that interfaces with the hypothalamus. ${ }^{11,12}$ Seizure propagation has a direct route to the hypothalamus $^{5,12}$ The concept of disconnection between the HH and hypothalamus is more feasible than excision of the $\mathrm{HH}$ for achieving seizure control while avoiding complications. ${ }^{5,12}$

Early reports of SRT mentioned that patients with small or medium-sized HHs were surgical candidates for SRT. ${ }^{4,13,25}$ A potential disadvantage of the SRT procedure is the use of multiple trajectories and coagulated lesions. ${ }^{16}$ However, we found no differences in outcomes between patients with giant and nongiant HHs. We have performed the unilateral approach on an ipsilateral attachment and the trans-third ventricular approach on a contralateral attachment, in a single stage, to avoid bilateral hypothalamic damage. Good seizure outcomes were achieved using this combination of approaches regardless of the numbers of lesions and trajectories. Our SRT procedure involves the use of a stereotactic multipurpose frame and arc system, which requires completion of the cranial sutures and should therefore be limited to patients who are 2 years of age or older. The only other restriction for our procedure would be a pedunculated type of $\mathrm{HH}$, although we have never encountered an epileptic patient with such a tumor.

\section{Surgical Complications}

Various surgical complications-including some that involved hypothalamic symptoms-were transiently observed after SRT. However, an increase in the occurrence of hyperthermia was the only significant complication. It was seen in the pediatric patients and may have been caused by the significantly larger numbers of trajectories required in the pediatric group. Immediately after SRT, transient neurological symptoms of supplementary motor area (SMA) syndrome were noted. Moreover, EFP of the side contralateral to the SRT surgery was oberved in this series. This postoperative neurological sign has not been previously reported, and it seems to be peculiar to the SRT procedure. Furthermore, we did not find EFP in our earlier series, ${ }^{9,12}$ although the numbers of trajectories and lesions were less than in our present cohort. We consider that postoperative EFP may be a supplementary motor symptom associated with transient hypokinesia of the contralateral limb after SRT. EFP is characterized by impaired activation of the facial muscles with emotion, without any corresponding impairment in voluntary mimicry. Laplane et al. ${ }^{15}$ reported three cases of contralateral EFP after SMA corticectomy. A transient motor disorder of the SMA is likely caused by inactivation of the network between the mediodorsal nucleus of the thalamus and the hypothalamus after SRT.11,23

There were only 2 cases of postoperative permanent pituitary dysfunction in our cohort, and they involved patients who had undergone repeat SRTs for bilateral attachment. Therefore, a bilateral SRT approach to bilateral attachment should be avoided. The rate of permanent complications in our study was lower than the 9\%-15\% incidence of significant long-term complications (including memory impairment, hemiparesis, and endocrine dis- 
turbance) after transcallosal and endoscopic resection or disconnection..$^{20}$ Although Gamma Knife radiosurgery has been considered much as safer than microsurgery, in one study, $10 \%$ of patients were reported to exhibit the adverse effect of poikilothermia. ${ }^{21}$ Although short-term memory loss is a transient symptom in $8.6 \%$ of patients after SRT, Rekate et al. ${ }^{22}$ reported that 3 patients $(7 \%)$ had transient short-term memory loss and $3(7 \%)$ had persistent memory loss after endoscopic surgery. Wait et al. ${ }^{24}$ reported persistent memory loss in $8 \%$ of patients after a transcallosal approach and permanent short-term memory loss in $8 \%$ of patients after endoscopic resection.

\section{Seizure Outcomes}

A single SRT was effective in approximately $70 \%$ of our patients. Moreover, the repeat surgeries were effective without increasing the rates of postoperative complications. Furthermore, SRT is a feasible additional surgical option regardless of any prior surgical treatment. To our knowledge, the seizure outcomes of our SRT method were better than those previously reported. ${ }^{20}$

The lower rate of freedom from non-gelastic seizures in adult patients is likely because non-gelastic seizures are caused by independent secondary epileptogenesis. Parvizi et al. ${ }^{18}$ also reported that patients with non-gelastic seizures were significantly older and had significantly longer duration of epilepsy than patients with only GS. Therefore, early surgery is important for the prevention of independent secondary epileptogenesis.

\section{Functional Outcomes}

Patients with comorbidities such as mental retardation, behavioral disorder, or precocious puberty had significantly larger lesions than those without these conditions, as previously reported. ${ }^{18}$ Our SRT procedure had no effect on precocious puberty. In addition, some patients experienced delayed precocious puberty after SRT. Although postoperative weight gain was observed in our series, the overall rate was lower than the rate previously reported in a case series involving adult patients treated with resection or radiosurgery. ${ }^{6}$ Thus, we consider that these delayed hormonal disorders likely reflect the natural history of the disease, because our procedure involved making a disconnection. Almost all patients achieved good functional outcomes with respect to cognitive development, with the exception of patients with severe intellectual and developmental disorders. Behavioral improvement generally required shorter recovery times than cognitive development. Although successful surgery of $\mathrm{HH}$ itself can treat epileptic encephalopathy,,$^{1,26}$ our SRT procedure based on the concept of disconnection of the epileptogenic pathway also stop epileptic "bombardment" of the thalamic mediodorsal nucleus and the resultant epileptic encepalopathy. ${ }^{11,12}$ On the basis of the functional outcomes observed after surgical treatment, we believe that surgical procedures should be considered after a shorter duration of GS history to prevent the development of epileptic encephalopathy.

\section{Conclusions}

The present SRT procedure is a minimally invasive and highly effective for treatment of HH. SRT involves a single surgical procedure applicable to all forms of epileptogenic HH. SRT procedures should be considered early after the definite diagnosis of $\mathrm{HH}$. Seizure freedom can allow functional recovery and development early in life for $\mathrm{HH}$ patients.

\section{Acknowledgments}

We thank Drs. Hiroatsu Murakami and Nobuo Satoh for their technical assistance. This study was supported in part by a Health Labour Sciences Research Grant, the Ministry of Health, Labour and Welfare of Japan and a Research Grant from the Japan Epilepsy Research Foundation.

\section{References}

1. Berkovic SF, Arzimanoglou A, Kuzniecky R, Harvey AS, Palmini A, Andermann F: Hypothalamic hamartoma and seizures: a treatable epileptic encephalopathy. Epilepsia 44:969-973, 2003

2. Calisto A, Dorfmüller G, Fohlen M, Bulteau C, Conti A, Delalande O: Endoscopic disconnection of hypothalamic hamartomas: safety and feasibility of robot-assisted, thulium laser-based procedures. J Neurosurg Pediatr 14:563-572, 2014

3. Choi JU, Kim DS: Treatment modalities for intractable epilepsy in hypothalamic hamartoma. Adv Tech Stand Neurosurg 39:117-130, 2012

4. de Almeida AN, Fonoff ET, Ballester G, Teixeira MJ, Marino R Jr: Stereotactic disconnection of hypothalamic hamartoma to control seizure and behavior disturbance: case report and literature review. Neurosurg Rev 31:343-349, 2008

5. Delalande O, Fohlen M: Disconnecting surgical treatment of hypothalamic hamartoma in children and adults with refractory epilepsy and proposal of a new classification. Neurol Med Chir (Tokyo) 43:61-68, 2003

6. Drees C, Chapman K, Prenger E, Baxter L, Maganti R, Rekate H, et al: Seizure outcome and complications following hypothalamic hamartoma treatment in adults: endoscopic, open, and Gamma Knife procedures. J Neurosurg 117:255-261, 2012

7. Fenoglio KA, Wu J, Kim Y, Simeone TA, Coons SW, Rekate $\mathrm{H}$, et al: Hypothalamic hamartoma: basic mechanisms of intrinsic epileptogenesis. Semin Pediatr Neurol 14:51-59, 2007

8. Fukuda M, Kameyama S, Wachi M, Tanaka R: Stereotaxy for hypothalamic hamartoma with intractable gelastic seizures: technical case report. Neurosurgery 44:1347-1350, 1999

9. Homma J, Kameyama S, Masuda H, Ueno T, Fujimoto A, Oishi M, et al: Stereotactic radiofrequency thermocoagulation for hypothalamic hamartoma with intractable gelastic seizures. Epilepsy Res 76:15-21, 2007

10. Kahane P, Ryvlin P, Hoffmann D, Minotti L, Benabid AL: From hypothalamic hamartoma to cortex: what can be learnt from depth recordings and stimulation? Epileptic Disord 5:205-217, 2003

11. Kameyama S, Masuda H, Murakami H: Ictogenesis and symptomatogenesis of gelastic seizures in hypothalamic hamartomas: an ictal SPECT study. Epilepsia 51:2270-2279, 2010

12. Kameyama S, Murakami H, Masuda H, Sugiyama I: Minimally invasive magnetic resonance imaging-guided stereotactic radiofrequency thermocoagulation for epileptogenic hypothalamic hamartomas. Neurosurgery 65:438-449, 2009

13. Kuzniecky RI, Guthrie BL: Stereotactic surgical approach to hypothalamic hamartomas. Epileptic Disord 5:275-280, 2003 
14. Kuzniecky R, Guthrie B, Mountz J, Bebin M, Faught E, Gilliam F, et al: Intrinsic epileptogenesis of hypothalamic hamartomas in gelastic epilepsy. Ann Neurol 42:60-67, 1997

15. Laplane D, Talairach J, Meininger V, Bancaud J, Orgogozo JM: Clinical consequences of corticectomies involving the supplementary motor area in man. J Neurol Sci 34:301-314, 1977

16. Mittal S, Mittal M, Montes JL, Farmer JP, Andermann F: Hypothalamic hamartomas. Part 2. Surgical considerations and outcome. Neurosurg Focus 34(6):E7, 2013

17. Munari C, Kahane P, Francione S, Hoffmann D, Tassi L, Cusmai R, et al: Role of the hypothalamic hamartoma in the genesis of gelastic fits (a video-stereo-EEG study). Electroencephalogr Clin Neurophysiol 95:154-160, 1995

18. Parvizi J, Le S, Foster BL, Bourgeois B, Riviello JJ, Prenger E, et al: Gelastic epilepsy and hypothalamic hamartomas: neuroanatomical analysis of brain lesions in 100 patients. Brain 134:2960-2968, 2011

19. Pati S, Abla AA, Rekate HL, Ng YT: Repeat surgery for hypothalamic hamartoma in refractory epilepsy. Neurosurg Focus 30(2):E3, 2011

20. Pati S, Sollman M, Fife TD, Ng YT: Diagnosis and management of epilepsy associated with hypothalamic hamartoma: an evidence-based systematic review. J Child Neurol 28:909-916, 2013

21. Régis J, Carron R, Bartolomei F, Chauvel P: Seeking new paradigms in epilepsy: stereotactic radiosurgery. Clin Neurosurg 59:59-69, 2012

22. Rekate HL, Feiz-Erfan I, Ng YT, Gonzalez LF, Kerrigan JF: Endoscopic surgery for hypothalamic hamartomas causing medically refractory gelastic epilepsy. Childs Nerv Syst 22:874-880, 2006

23. Taber KH, Wen C, Khan A, Hurley RA: The limbic thalamus. J Neuropsychiatry Clin Neurosci 16:127-132, 2004

24. Wait SD, Abla AA, Killory BD, Nakaji P, Rekate HL: Surgical approaches to hypothalamic hamartomas. Neurosurg Focus 30(2):E2, 2011
25. Wang W, Wang W, Guo X, Zeng Y, Jiang X: Hypothalamic hamartoma causing gelastic seizures treated with stereotactic radiofrequency thermocoagulation. Epileptic Disord 11:333-338, 2009

26. Wethe JV, Prigatano GP, Gray J, Chapple K, Rekate HL, Kerrigan JF: Cognitive functioning before and after surgical resection for hypothalamic hamartoma and epilepsy. Neurology 81:1044-1050, 2013

27. Wilfong AA, Curry DJ: Hypothalamic hamartomas: optimal approach to clinical evaluation and diagnosis. Epilepsia 54 (Suppl 9):109-114, 2013

\section{Disclosure}

The authors report no conflict of interest concerning the subjects or methods used in this study or the findings specified in this paper.

\section{Author Contributions}

Conception and design: Kameyama. Acquisition of data: Shirozu, Masuda, Ito, Sonoda. Analysis and interpretation of data: Kameyama, Shirozu, Sonoda. Drafting the article: Kameyama. Critically revising the article: Kameyama, Shirozu, Masuda, Ito, Sonoda. Reviewed submitted version of manuscript: Kameyama, Shirozu, Masuda, Ito, Sonoda. Approved the final version of the manuscript on behalf of all authors: Kameyama. Statistical analysis: Sonoda, Akazawa. Study supervision: Kameyama.

\section{Correspondence}

Shigeki Kameyama, Department of Functional Neurosurgery, Epilepsy Center, Nishi-Niigata Chuo National Hospital, 1-14-1 Masago, Nishi-ku, Niigata 950-2085, Japan. email: kameyama@ masa.go.jp. 